source of loss and confusion to potato producers and merchants. The synonym committee of the institute, consisting of experts both scientific and practical, makes an annual report which declares which of the varieties entered for the immunity trials under new and distinct names prove to be identical with varieties already in the market.

During their visit the King and Queen were able to see the normal work of the Official Seed Testing Station at progress in most of the laboratories. The exhibits of cereals and potatoes arranged in two of the laboratories enabled the visitors to realise the scope of the other branches of the institute's work. Their Majesties were keenly interested in everything that was shown to them, inquiring minutely into the processes of seed-testing, and paying special attention to the methods of potato-breeding and the measures taken to check the spread of wart disease. At the close of their visit they expressed to Sir Lawrence Weaver their complete satisfaction with all that they had seen and their admiration of Mr. Horder's planning of the buildings and the arrangements made to secure the efficiency and comfort of the staff.

\title{
Chemical Reactivity and the Quantum Theory.
}

By Dr. Eric K. Rideal.

$\mathrm{T}^{\mathrm{H}}$ $\mathrm{HE}$ recent discussion held by the Faraday Society on modern developments in the theories of catalytic chemistry gave rise to an important debate concerning what has been termed the radiation theory of chemical action. It is now almost generally accepted, both by the protagonists and by some of the opponents of this theory, that molecules of the same species in a reacting system may differ from one another in what is termed chemical "activity." Thus in a mixture of hydrogen and oxygen a certain fraction, both of the hydrogen and of the oxygen molecules, are "active." Collision between active molecules of the two species results in chemical combination; collision between inactive molecules produces no chemical change.

It is further argued by the supporters of the theory that true monomolecular chemical reactions exist, e.g. the conversion of allotropes, the dissociation of a diatomic gas, or the decomposition of substances like phosphine or ammonia; consequently, as pointed out by Perrin, "active" molecules must exist per $s e$, and reaction is not the result of a particular kind of directive collision, or, indeed, of a collision taking place at some particular phase of the molecular vibration; the decomposition of phosphine thus finds an analogy in the disruption of radium.

Granting that this assumption is correct, attention has to be directed to the source of energy of activation. The opponents of the theory, who go as far as to admit the validity of the first postulate, affirm that this energy resides within the molecule itself, and may possibly be identified with the "null punkt " energy at absolute zero.

The supporters of the radiation theory adopt the hypothesis that the energy of activation is acquired from the circumambient radiation, and that in consequence all reactions are in the broadest sense of the term photochemical. The energy supplied to one molecule so as to make it "active" to undergo the given reaction, whether it be explosion or combination with another active molecule, is assumed to be supplied by radiation of a particular frequency, and in amount equal to $h v$, where $h$ is Planck's constant.

For all the ordinary chemical reactions the amount of energy of activation to be supplied, as calculated by application of the fundamental equation of Arrhenius to the temperature coefficient of the reaction, is sufficiently small as to permit of the utilisation of quanta in the infra-red portion of the spectrum; for some reactions, however, visible or ultra-violet light will be necessary, whilst for accelerating the rate of change of radio-active decomposition ultra-X-rays would be required.

The equation of Wien on radiation intensity, and of Arrhenius on the temperature coefficient of chemi- cal reactions bear a formal resemblance to one another, and it is not doubted that the same fundamental properties of matter and of radiation account for the similarity. It is further admitted that the quantum theory of Planck, applied by Bohr to the internal structure of the atom, is likewise valid in many physical and chemical operations, such as calculation of the latent heats of change of state, the heats of formation of chemical compounds, including heats of ionisation and the photoelectric effect. More recently the quantum theory has been applied with success to a general study of reaction kinetics, and it is now evident that there is no essential difference between a typical monomolecular chemical reaction, such as the decomposition of phosphine, and a physical reaction like evaporation. It cannot be doubted that both physical and chemical forces are identical in their nature and also in their mode of action.

The opponents of the theory admit these premises, but see no reason to assign to radiation the important rôle given to it by its adherents, and prefer to attribute the two phenomena to some common, but as yet unknown, property, giving rise to these apparent similarities.

The supporters of the theory point out that in fact many photochemical reactions do exist, and, thus admitting the possibility of the direct action of radiation on matter in causing both physical and chemical change, there is no reason why this property should not be universal.

In the development of the theory in its quantitative aspects, however, certain difficulties have arisen necessitating a modification of the simple theory originally proposed; thus the rate of decomposition of phosphine has been accurately determined over a wide range of temperature, the frequency of the radiation necessary to bring about its decomposition calculated from the reaction temperature coefficient, and the amount of energy flowing into the reaction chamber.per c.c. per second at the observed temperature calculated from Planck's law. It has been found that there is not enough energy supplied by radiation to account for all the explosions actually observed.

To account for this serious discrepancy several hypotheses may be advanced. Thus we may assume that during the explosicn of one phosphine molecule, which has already been activated by the absorption of one quantum of radiant energy, energy is radiated and absorbed by another molecule or by other molecules. Since it is not permissible to assume absorption in fractions of a quantum, it is necessary to adopt an hypothesis of activation of the phosphine molecule by a number of smaller quanta (infra-red) 
instead of one big quantum; this in turn seriously affects the logic of the deduction of the energy of activation from the temperature coefficient of the reaction.

Again, we may assume that when there is a continuous drain on one portion of the spectrum by absorption of the light of one particular frequency, the rest of the spectrum undergoes a continuous redistribution of the energy involving an increase in the absorbed radiation density and a decrease in the density of the radiation of greater and smaller wavelength. To account for such an hypothesis we must assume that there is some mechanism for the absorption of these rays in order to effect the redistribution, the purely monochromatic character of the reaction being thus lost, and a parallelism between these thermo- and photo-chemical reactions no longer exists. It is, of course, evident that such a redistribution of the energy does not take place when a reaction is illumined with ordinary visible light, since definite absorption bands are noted, and the rest of the energy either passes through the reaction chamber or is scattered from the molecule surfaces. A third hypothesis involves the assumption that the radiation density inside the actual molecules themselves is the important factor, and one which is greatly influenced by the refractive index of the molecule; computations on these lines lead to high values for the refractive index of the region inside the molecules which await other independent confirmation.
A second difficulty has been raised by a study of hydrolytic operations, e.g. sucrose; the temperature coefficient of the reaction indicates an activating frequency in the infra-red portion of the spectrum. Illumination with bright sunlight should cause a very great increase in the reaction velocity; no perceptible effect is actually observed. It is, however, possible to attribute the comparative inertness of these reactions to the strongly absorbent character of the environment to light of long wave length; and it has been suggested that a study of the reaction velocity in thin films under illumination might lead to positive results.

Of significance is the fact that the substance for which the activating frequency has been calculated from the temperature coefficient frequently shows no absorption band in that region. If the hypothesis be adopted that the activating frequency calculated in this manner is only a mean value, i.e. the possibility of activation in stages be envisaged, we are confronted with a difficulty in calculating the reverse operation, viz. the reaction velocity from a knowledge of the complete spectrum of the reacting system, since we have no information as to the manner of the distribution of the partially activated molecules.

The debate served clearly to emphasise the relationship of the quantum hypothesis to chemical action, and the fact that the radiation theory was not entirely convincing, but, on the other hand, it certainly contains the germ of the solution to the problem of the mechanism of the interaction of matter and radiation.

\section{The Teaching of Geography.}

I $N$ opening the discussion on the teaching of geography at a joint meeting of the Sections of Geography and Education of the British Association at Edinburgh on September 9, Mr. G. G. Chisholm laid stress on the physical basis of geography, but urged the importance of regarding the physical agencies not so much as changing the face of Nature as influencing the distribution of man and his activities. Mr. Chisholm pointed out that geographers have now reached a considerable measure of agreement in the work included under the head of geography. That agreement marks a step in the better recognition of geography in the educational curriculum. At a later stage in the discussion Dr. H. R. Mill dwelt on the urgent necessity of quantitative work in geographical research, and pointed out the enormous field of study which this opens.

Sir Richard Gregory spoke of the position of geography in relation to other science subjects in the school curriculum. He advocated a course of general science as more useful for a general education than the beginnings of heat and light and the laws of chemical action for pupils up to the age of sixteen. A course in geography for all pupils up to that age would at the same time provide the unifying principle for all the science work, bringing it into relation with the activities of man. After such a course it would be equally easy for pupils to specialise in mathematics, physics, chemistry, or geography. At present there tends to be a gap in geographical teaching between school and university work, because few schools have geography teachers capable of carrying the subject to a standard equivalent with the teaching in chemistry and physics.

Mr. W. H. Barker deprecated the tendency to divide studies into watertight compartments, and insisted that the teaching in geography must be given, not by the science master, but by a geography specialist who by his training is fitted to bring out the unifying No. 27 I 2, VOL. IO8] influence of the subject. Geography serves to unite the two main groups of subjects, natural sciences and humanities, and therein lies its great educational value. To reunite the specialisations is the function of the geography teacher.

Some of the difficulties of getting adequate recognition of geography in the university curriculum were pointed out by Dr. Rudmose Brown. The rigid division of studies into the faculties of arts and science is only slowly breaking down, and, in consequence, geography has a fight to find its true position. The geographer has a definite outlook, and his subject is the same, no matter in which faculty it is placed. The narrow conception of science as being confined to the so-called natural sciences must give way before geography can find adequate recognition. Meanwhile, the practical result of dividing knowledge by a rigid line of demarcation is reflected in the difficult task of giving students of geography the wide outlook that the subject requires.

A plea for the value of geography in historical study was made by Prof. R. K. Hannay. He complained of the non-geographical attitude of many historians, and urged that students of history should follow courses in geography. Prof. J. W. Gregory, in em. phasising the scientific basis of geography, thought that it should be included in the science faculties of universities, but did not disparage its inclusion among arts subjects. While there has been much improvement in geographical education in Scotland in recent years, the subject still suffers from neglect and failure to take its due portion in education. This is most noteworthy in secondary schools. Dr. F. Mort was hopeful of the position of geography in Scottish schools, and quoted figures to show the increased numbers taking advanced work in the subject and taught by specially trained teachers. Prof. J. A. Green regretted that much school geography was above the heads of the pupils, the teacher not in- 